\title{
An Insight into Patients' Perspectives on their Participation in Medical Decision Making in Diabetes Mellitus Care in Malawi.
}

\section{Martha Makwero ( $\square$ mmakwero@cartafrica.org ) \\ University of Malawi}

\section{Adamson Muula}

University of Malawi

\section{Felix Anyanwu}

University of Venda

Jude Igumbor

University of the Witwatersrand

\section{Research Article}

Keywords: Diabetes Mellitus, patient participation, patient involvement, shared decision making

Posted Date: January 13th, 2021

DOI: https://doi.org/10.21203/rs.3.rs-141060/v1

License: (a) (i) This work is licensed under a Creative Commons Attribution 4.0 International License. Read Full License 


\section{Abstract}

Background: Patient participation in their own care has potential to improve experiences and responsiveness in chronic disease situations. Patient participation is a basic tenet for a patient centred care experience. However, the documented experiences show that patient participation in care decisions making is wanting. As Malawi strives to imbibe and institutionalise the concept of patient centred care, it is important to examine the experiences of patients regarding participation in their care as this may provide evidence to support policy implementation strategies on the same.

Aim: The study therefore sought to describe DM patients' experiences of participation in shared decision making in Malawi.

Methods: This qualitative exploratory study involved patients attending DM clinics in four public health facilities in Southern Malawi. Using SHARE framework, we analysed data from 15 in-depth interviews and two focus group discussions.

Results: From the perspective of DM patients, this study elucidated that participation in care was largely lacking in clinical encounters. Some of the prominent gaps identified in the interactional process of care included: i) unfavourable ambience for participation, ii) insufficient bidirectional informational exchange and iii) inconsideration for patients' preferences and values, iv) paternalistic power tendencies and, v) lack of evaluation of decisions made.

Conclusion: The study highlights gaps in the practice of patients' participation in medical decisions. Patients' reproof of paternalistic models of care confirms that transforming medical education through curriculum review for providers may be a good starting point. The review should focus on the content and effective delivery of competencies relating to provider roles and practices on patient participation.

\section{Background}

Investing in individual or collective patients' participation in medical care decisions is a fundamental step in enhancing a patient centred care (PCC) experience (1). Patient participation during clinical encounters is considered a basic tenet for achieving patient empowerment and enabling patients to be active recipients of their care (1-3). Essential elements in patient participation involves active patient engagement and involvement that facilitate care responsiveness and respect for patient values (4). In practice, patients' experience of participation in medical decisions has been documented to lack deserved consideration; limited often limited to informing patients and expecting them to comply to providers' care plans $(5,6)$.

Patient participation in decision making refers to interactional elements between the patient and the provider; in which patients provide information, asks questions and shares experiences that contribute to the exploration of problems, identification of management options that meets patients' needs, preferences and priorities of care(7). Genuine participation thrives in a mutuality and trusting environment where both parties perceive a conducive space to express themselves in a dialogue (7). Patient participation is a complex phenomenon that extends through a continuum from patient manipulation to total control (1). Thus, optimal participation culminates in collaborative patient management plans, a proactive patient -provider dyad that has potential to 
actively engage, empower and enable patient in reaching their personalised goals; with the provider as a resource $(2,8)$. Hence, patient participation facilitates provision of care that meets at the juncture of patient preferences, provider experiential practice and research evidence.

As a process of care, patient participation in decision making enhances positive experiences of care $(2,9)$. Particulary in the chronic disease journey, participation assists in treatment goal setting facilitating medication adherence, and lifestyle modification through enhancement of patient self-efficacy for self -care $(2,10,11)$. Thus patient participation becomes key in DM patients as they have to constantly make decisions to accommodate their complex individual situations, recurrent hospital visits, the disease, its treatment and goals $(8,12)$.

Involving and engaging patients has been shown to improve clinical decision making, provider- patient concordance in making and achieving personalised goals of care(1). Further, participation improves patients' disease knowledge and their appreciation of risk, encourages patients' to have realistic expectations of care, enhances patient autonomy that facilitates better self-care and, ultimately experiences of satisfaction with care (1-3). Patient participation has also been associated with improved biomedical outcomes such as glycaemic control $(1,3)$. In a study by Wang et al on type 2 DM patients, it showed increased odds of improved better glycaemic control if the patients perceived being involved in care decisions; and that decisions were made collaboratively (3). Even though only $10.3 \%$ and $39.3 \%$ of the patients in this study preferred "patient led" and "shared" decision making respectively, it highlights that patients' participation and the resultant collaborative decisions have potential to improve patient clinical outcomes too $(15,16)$.

Castro et al highlight the following dynamic processes in the patient-provider interaction as a key antecedent to patient participation. The processes include:- i) deliberate effort to invite and encourage the patient or their proxies to participate in a supportive dialogue; facilitated by therapeutic relationship, reduced the power gap, ii) engage in meaningful dialogue about the disease and, the individualised treatment options available, and iii) supportive interactional environment that aims to activate and empower patients to actively participate to the extent they desire; refraining from merely validating providers' agenda (4). The process further requires acknowledgement and appreciation of patients as experts of their disease in a trusting and respectful atmosphere(5). Ultimately, the process has potential to engender a well informed and activated team of patients and providers that is better placed to meet the demands of DM care(6).

While variations in the extent to which a patient wishes to participate in care across and in between encounters has been reported in literature, most patients are reported to prefer some level of participation(7-10). Elwyin et al adds that there is generally very minimal objections about patient involvement amongst different patients groups(11). In spite of stated outlook, the practice of patient participation is marked with failings(7-10). An interplay of patient, provider, system and contextual factors have been reported to affect individualised levels of participation in care at interactional (micro), institutional (meso-) and system (macro-) levels(4, 12). At the patient-provider interactional level, some of the factors known to influence its dynamics include; patient socioeconomic profile such as education status and health literacy, past experiences, their views and values. Additionally, providers' communication skills, attitudes towards shared decision making and conflicting roles have been known to affect the level of patient participation $(4,5,7,9)$. For instance, Shortus et al report that providers often are conflicted as to whether to pursue patients' personalised goals or biomedical targets(7). Thus, they are often uncertain to what extent to engage their patients towards personalised goals. While at 
institutional level, it may include factors such as interactional time constraints, and at systems level, contextual issues such as an authoritarian socio-political context(9). For example, patients with authoritarian sociocultural backgrounds have been reported to have lower the likelihood of initiating engagement with a medical authority or to participate in their own care $(9,10)$.

The attention given to user perspectives is increasingly a becoming fundamental to the quality service delivery discourse in Malawi chronic care settings(8). As we seek for more individual or collective participation in chronic patients such as DM, this narration of patients' perception of their experience of participation of care becomes imperative as they have potential to influence strategies towards improved practice in patient participation in decision making $(9,13)$. The narrative in this paper therefore seeks to describe and examine the depicted experiences of participation in decision making, its prominent gaps. The findings have potential to facilitate realistic debate on how to facilitate the needed participation in care among DM patients.

\section{Methods}

To address our aim, we used the qualitative exploratory inquiry approach which enabled a methodical scientific discovery of understandings and perceptions from participants' experiences of care $(14,15)$

\section{Participants}

We studied the narratives of DM patients accessing care at the DM clinics. Patients who have been accessing care for more than six months in the stated facilities were included in the study. We recruited a total of 37 patients.

\section{Data collection}

Data was collected using un-structured questionnaire (see attached supplementary file). We conducted 15 indepth interviews and two focus group discussion of 11 participants each. All interviews were conducted in the local within the clinic building by the first author. Narrated experiences of participation in care decisions were explored as part of a larger study that sought to find out the meaning of patient centred care. Apart from exploring perceptions about patient centred care, participants were further probed to narrate their experiences around their participation in common decisions around their care.

The study was conducted in four DM clinics at designated public hospitals in southern Malawi. The public sector was purposively chosen because they are the largest healthcare provider in Malawi (16). Thus insights obtained have the potential may reflect what happens in the significant care sector. The study was conducted from September to December 2019.

Credibility, transferability and conformability measures in line with Shenton et al were safeguarded in this study 19). We ensured credibility by triangulating information from in-depth interviews with focus group discussions. Transferability of the results was enhanced through diversifying the patient profiles within the DM group studied. A thorough description of data collection methods and its quality assurance checks allowed dependability of the study to obtain similar results if it were to be repeated elsewhere. Again, as a way of quality check, two random transcripts were coded by the first and the third authors concurrently. Differences in coding were discussed and resolved. Conformability was achieved through counterchecking the codes by 
another individual that is from a different background to help mitigate the effect of researcher's prior predispositions that might have affected the analysis.

\section{Data analysis}

The data analysis process started during data collection in the field by noting and exploring further on the themes that came out recurrently. Following the data collection, translation into English and transcriptions was conducted. Later, we imported the data into NVivo software version 11 for analysis.

The analytical process involved familiarisation, coding, mapping data against the SHARE framework (18) which highlights the gaps in the practice of patient participation in care. Familiarisation with the data set was done through reading transcripts repetitively. Through this step we identified and noted the recurrent themes deductively from participants' accounts. Saturation of responses was arrived after 13 IDIs. Themes were subsequently grouped manually to come up with the initial codebook; which was iteratively refined further through further discussion in liaison with the third author. Exemplar extracts were then mapped against the codes.

\section{Results}

\section{The process of participation in decision making}

The study presents results of DM patients' experiences in their involvement of care. To highlight the salient gaps in the process of participation, the SHARE framework has been used to describe the themes we identified to highlight gaps and possible underlying reasons (18). The framework demonstrates essential steps in shared medical decision making namely; i) Seeking patients' participation, ii) Helping patients to explore and compare available treatment options, iii) Assessing patients preferences and values, iv) collaboratively Reach a decision, iv) Evaluate the decisions made. Thus, the gaps have been organised in the following themes : i) unconducive environment for collaboration ii) Unsatisfactory information exchange iii) Inconsideration of patient values and preferences iv) Provider paternalistic tendencies to participation v) Poor assessment of decisions made.

\section{Theme 1: Non-inviting atmosphere for collaborative dialogue}

Most DM patients felt that there was no space or that the ambience is unreceptive for participation in shared decision making; stating that providers do not cordially engage patients into discussion about their disease, the treatment or matters that concern them.

'Sometimes it is bad. A patient should be asked and should be allowed to give his/her suggestions, that way it would be easier for him/her to follow the decisions made. So it is very important to inquire from us first.'(Male participant 2 rural FGD)

'Their reason [for not discussing with me] being I am from the village as such I cannot manage to buy insulin. I was just listening to them. "No, prescribe tablets for him. Tablets are much cheaper." And then I asked them questions. "What exactly are you saying? Who told you this? Did you ask me so that you may hear my own side? Because the insulin is for me and not for you. I have left my home to come here and the first thing you should do is to inquire more about me and I should tell you' (Male participant 5 rural FGD) 
This excerpt alludes the lack or failure to engage patients through a dialogue in order to understand the problem and explore or offer management options collaboratively. Some reasons for this failure is assumption of patients' low medical literacy or low social economic status.

Some patients also expressed that they fail to contribute to the discussion because the ambience is perceived unfavourable. The patients therefore refer to the patient -provider interactional experience as fearful and dismissive during attempts to participate in SDM. As a result patients assume a submissive and unknowledgeable role; hence, passive recipient of care.

'What scares us is, whenever you are talking too much and suggesting ideas to the doctor, they just ask you one question, "Are you the doctor? Just go and do whatever you are told." And then we don't have anything to say' (female participant 9, urban FGD)

Patients' feeling of fear is perpetuated by the possibility of being denied the service intended for that day if they ask questions in pursuit for engagement. Again some patients felt that it is culturally inappropriate to engage with their provider in exchange of ideas. They perceive that their role is to gratefully receive the intended package of care without question.

'My suggestions [are] if you are patient and you are meeting the doctor, there is no way you can be exchanging ideas. The doctor just tells you that depending on your results, follow this, this and this. I will prescribe these drugs for you. I don't think a patient can have the audacity to be telling the doctor what to do as if you know about health. You just listen and take whatever the doctor has told you. (Participant 5 urban FGD)

\section{Theme 2: Unsatisfactory informational exchange}

Patients 'experience was that sound patient involvement is precluded by insufficient informational exchange between the patient and the provider during the encounter. Further alluding that there is hardly any attempts for informational exchange to facilitate co-construction of sound propositions to base management decisional options upon.

'However there has to be a good motivation, you [the provider] should ask questions, I should answer. I should ask questions and you should explain to me that for your problem to be over, you should do this and I should try that. "How are you feeling now?" "Everything is well." By doing that we have helped each other questions and seeking clarifications from patients.' (Female participant 2 FGD urban)

'But I still insisted that it would have been better if they had asked me first. "How do you feel? From the way you are feeling, can you try this?" "Your friends already tried but they failed and they recommended me to be on insulin." Such scenarios can bring sour relationships between providers and patients. Sometimes it happens and it happened to me at XXXX [hospital].'(Male participant, IDI rural)

The quotes also highlight patients' aspiration that their provider should be the one to initiate the informational exchange. Patients perceive the lack of informational exchange to jeopardise efforts for provider- patient therapeutic relationship building. Unfortunately, the experience that providers hardly allow time for informational exchange is widespread as this patient reports. 
'There are some provider[s], especially doctors, before you finish explaining, they have already written in your book so what can you do? Can you continue talking with them? Doctors are the ones who are supposed to listen to our complaints but most of them dispose us off even before we finish talking'. (Female participant IDI urban)

Patterns of hurried and dismissive interactions that do not favour bidirectional information exchange are testified in this study. While complaining that they are not listened to, patients make reference to the significance of bidirectional informational exchange. Further, they allude that; whereas providers bring technical knowledge, patients have potential to offer experiential insights into their disease and management. Thus, patients confirm their valuable role as experts in their disease and that they need to be allowed time to contribute to the discussion table.

'Just imagine a fresh doctor coming from College of Medicine trying to tell a patient who has been diabetic for 18 years. Who knows much there? It is us patients. That is why there is a need for that good doctor-patient relationship. Just because they learnt theory doesn't mean that they know everything. We are the ones who know much because we are the patients and they are supposed to listen to us. They should tell us what they learnt, the new technologies coming in but we should also tell them about our disease because we have an experience of the condition and by doing that we will help each other.' (Female participant 7, urban FGD)

\section{Theme 3: Inconsideration for patients' preferences, values and capabilities}

Following informational exchange; the patients felt that the provider ought to explore patient's preferences, capabilities and values.

'Ok good. According to you, can you manage to access these type of drugs?" "Yes. I am employed. I have things in my house. I can always sell them [things/belongings in my house] to buy drugs" "Really?" The provider should be writing all that. Indeed this patient is able to access drugs. But just making decisions without the knowledge of the patient sometimes it becomes a problem.'(Male participant 5, FGD rural)

Again, its shows that that patients yearn to take some control of their disease as this may reduce the congestion at the hospitals as this patient alludes

'We should be given that opportunity to be controlling ourselves and only come when something is wrong [....] There are a lot of patients and still others are on their way coming. So it will would really be of help. (Male participant 6, urban FGD)

\section{Theme 4: Paternalistic power inclinations}

Provider authoritarian tendencies in medical decisions were reported by patients and accepted as normal. Most respondents believed that they cannot collaborate with their providers; as they feel powerless to engage or challenge a provider authority. Due to lack of a collaborative opportunity, they feel obligated to put their trust in their provider, therefore, largely delegate decisional autonomy to their providers.

It is very difficult to have an opportunity to sit down with the doctor and discuss because mostly they feel like those issues should be left with the researchers and the diet which they have given us is ideal. So I don't think 
that even if we sit down and discuss, it won't change anything.' (Male participant, IDI urban)

The excerpt further highlights that patients do not actually believe that their voice would genuinely matter in the dialogue. Thus, even when they do have alternative options, or do not agree with a specific treatment option, their view points are unlikely to be taken seriously. As such some patients choose not voice out their opinion. Because patients feel powerless to negotiate with the provider, the clinical interactions are thus referred to as superficial as this female patient confesses:

'You should discuss properly. In a good relationship you should ask how you are feeling, anything beyond that they say you want to be the doctor. We have no control on other part. Our interaction is on the surface'. (Female participant, IDI urban)

While, the perception that patients' opinion is not substantial in the discussion is recorded, it is fuelled by the local socio-cultural notion a patient cannot confront the provider on issues the patient does not agree with for fear of appearing rude. Thus, in trying to conform to social norms, patients approach the interaction with a disempowered, blindly trusting perspective.

'You cannot give out your suggestions to doctors because they are the doctors. We trust whatever they say because they are doctors. It's the same as being in church. The pastor may be preaching and you just listen without saying a word. Maybe when you go out, that is when you may tell the pastor that you did not agree with whatever he was preaching. Similarly in the consultation room. You are with your doctor just the two of you but you wait till you go outside and then start complaining to other people what the doctor did to you instead of confronting the doctor in the room because you are afraid that the doctor might think you are being rude.'

(Female participant 5, urban FGD)

'So whenever we come here, we take the doctors as our help. We trust whatever they say.' (Female participant 2, urban FGD)

The extract highlights the effect of socio-cultural propensities affecting the interactional norms around patient participation in shared decision making. Whether the specified trust in the provider is genuine or not remains unclear.

Notably patients seem be aware of the providers' unspoken cues around providers' paternalistic inclinations and perceived reluctance to involve patients in care as highlighted below.

'..when you start asking the doctor too many questions, they leave you because they assume that you know everything. It is just out of luck when they write a prescription for you and that is why we are resilient to answer back. You can even tell that this doctor is angry at me and you don't ask any more questions. You just wait for the prescription and then off you go. We should say the truth, a patient should not be wise more than the doctor. Just listen to whatever s/he is saying even though s/he is wrong.' (Female Participant 9, FGD urban)

The reaction to the unspoken cues seems to propagate fear and further support the paternalistic culture. However, patients still appeal for collaborative dialogue as presented in excerpts below that highlights the plea for an opportunity for feedback. 
'Doctor, this is what has been going at the hospital and of course it is a good thing but also we have these challenges. The challenges should be sorted out." They should hear our plea and they would sit down to find best solutions for the challenges.' (Male participant 6, rural FGD)

'We want our problems to be heard and solutions should be implemented through the leadership of the facility. (Male Participant 5, rural FGD)

\section{Theme 5: Evaluation ofe decision made}

In a far as patients are concerned, evaluation for support and buy-in of some decisions made during the encounter is lacking.

"The doctors just saw that I was on insulin but they did not inquire why I was on the insulin. So sometimes providers can make a decision of which a patient is not in support of and the patient says it is better to die than to follow what the providers are saying. (Male participant 2 rural FGD)

Lack of mutual assessment of decisions made and their respective propositions is reported to be counterintuitive. Some decisions are reportedly not practical to the patient's life style as such are unlikely to be implemented thus; having detrimental impact on set goals of care or quality of life.

\section{Discussion}

In this study, we examined narratives of experiences of participation in medical decision care in DM patients and it exposed some gaps associated with patient participation practice in medical DM clinical encounters. This paper discusses the gaps including: i) unfavourable ambience for participation, ii) insufficient bidirectional informational exchange and iii) inconsideration for patients' preferences and values, iv) paternalistic power inclinations and, v) lack of evaluation of decisions made.

\section{The non-inviting atmosphere for collaborative dialogue}

Patients' experiences of a non-inviting ambience to participation in medical decisions is significant in this study. Cubaka et al, in their study describe that in a truly collaborative patient -provider interaction, inviting patients to a discussion is preliminary and core. There is evidence suggesting that patients are likely to participate if they are solicited, perceive a conducive environment where mutuality thrives, and genuinely feel safe and empowered to participate $(2,13,20)$. The patients in our study however describe dynamics of interactional process as fearful and dismissive; thus precluding them from engaging the providers in care even when they desired to or felt capable to.

Patients reported to perceive implicit anger from providers when attempts are made to engage with providers in a dialogue. Patients further presumed that the providers' anger would result in being denied a good service. Thus patients opt to assume subservient and unknowledgeable roles in order to obtain the care for the day(12). In a study by Ekdahl et al on elderly patients, they reported patients' passivity in relation to decision making. However, it was found that the passivity was related to fear of their providers reaction if they engaged in asking questions.(14) The stated experience highlights a failed therapeutic patient -provider relational ambience where patients do not feel safe enough to contribute or question about their care $(10,15)$. 
While the patient -provider power imbalance seems inevitable in clinical encounters, it is a reported drawback for lack of patients' participation $(9,10)$. Due to socio-political predispositions, patients in this study perceive that providers are a superior and unchallenged authority; and that their role should be a subservient, grateful recipient of care. Even though few patients reported insistence to be consulted about decisions made, the majority expressed lack of power and assertiveness to request participation. The lack of assertiveness can be corroborated in a study by Peek et al who studied patterns of decision making among DM patients from an ethnic minority. They noticed that patients' low medical literacy, poor socio-economic status (SES) and unawareness of their decisional freedom were some factors associated with low confidence to request for participation in decision making in ethnic minority groups(12,16). Additionally, that there was perceived power imbalances tilting against the patients; that was exacerbated when services are free and patients feeling that their health care is a privilege and not a right(16). Further, the imbalance left their providers to assume that they had inadequate capacity for meaningful engagement $(10,16)$. Thus, these studies highlight the need for provider attempts to level the power balance through a cultural shift that allows providers to acknowledge that patients are also experts in their disease $(9,13)$. Then, regardless of patient factors, solicitation of patients for participation and creating a participative ambience ought to be the provider's initiative.

\section{Inadequate reciprocal informational exchange}

Insufficient bilateral information sharing between the provider and the patient is apparent in the narrated experiences. Most patients in the study yearned for an opportunity for dialogue with their provider about their illness, its treatment and available options. Reciprocal information exchange is recorded as mandatory if patients are to engage meaningfully in medical decisions $(10,20)$. Factors deterring informational exchange include limited time, provider attitude, perception of low medical literacy, socio-cultural tendencies that perpetuate patient passivity $(11,20)$.

Often, interactional time constraints has been cited as a deterrent to satisfactory dialogue; perpetuating hurried and dismissive interactions 10,11). In a systematic review of 38 studies on sharing medical decisions by Thompson-Leduc et al, it showed that time constraint was cited as a deterrent to SDM in 22 out of the 38 studies (24). This confirms that interactional time is often limited; thus, a deterrent for adequate informational exchange. However, in a study done by Karel $\mathrm{M}$ et al on veteran mental health patients, they showed that the additional interactional time spent if the provider attempted to allow patients to participate in decision making only increased by three minutes (25). They further alluded that the practice of allowing patient participation is possible and cost-beneficial. Within the time constrained settings such as Malawi, the perception that information sharing is time consuming may seem legitimate $(11,26)$. However the gains from informational exchange have potential to improve patient-provider concordance, patient responsibility for self-care and trust. Additionally, potential to reduce unwarranted retreatments, and tilt power balances towards the patient $(13,22)$. Thus, the cost-benefit of the extra interactional time ought to be weighed in light of the resultant paybacks such as self-care, patient satisfaction and improved quality of life. We therefore suggest future studies on costbenefit analytical studies that would elucidate the cost averted with adequate informational exchange that results in patient enablement and empowerment.

Presumed readiness and low medical illiteracy is also a recognized deterrent to patient participation $(3,10,17)$. Patients in this study believe that their providers assume they are not ready or have insufficient capacity to 
engage in meaningful informational exchange. However, patients indicated they had enough capacity and experiential insights which they would love to share with the provider towards identification of the problem and exploring possible solutions. The experience is similar to a study by Ekdal et al confirms that providers perceived or were not sure to what extent their patients are ready and have capacity to participate in a dialogue $(14,18)$. The reviews therefore encourage explicit communication to establish concordance as to what extent their patients are willing and have capacity to engage on in decisions. Regardless of medical literacy level, patient's preferences of participation is to be explored. After all, Elywin et al record that most patients do not necessarily oppose participation in care rather it's the extent that matters (7). It is through the explicit dialogue that the individual patient desired level of participation will be delineated and those willing but have low capacity will be identified.

\section{Inconsideration for patients preferences, values and capabilities}

We notice that patients express lack of being acknowledged as experts in their own disease; asserting that providers disregard of patients' opinion. Consequently; patients feel uninvolved in exploring solutions to their own problems. The need to be acknowledged as experts can be confirmed in Lyles et al's editorial, who reported that patients yearned to feel that they have power to give their opinion and that it matters; that their capacities can be incorporated in care plans (19). Such a stance is achieved when the ambience is marked with genuinely trusting relationship. Castro et al in his narrative further admonishes paternalistic tendencies, acknowledging of patients expertise and capabilities in care as an attribute towards patient empowerment(4). Thus, inherently, individuals have the capacity to decide what is best $(4,26,27)$. The acknowledgment of patients' expertise has potential improve patient responsibility, satisfaction, and concordance.

\section{Paternalistic power inclinations}

Patient enablement is regarded as a critical and ultimate goal to PCC provision; tilting the power balances towards the patient $(4,22)$ Empowerment follows from deliberate efforts to invite patients to a discussion table and information sharing that leads to co-exploration of treatment options rather than validation of provider suggestions. Further, acknowledging and enhancing patient competencies; thus improving self-efficacy (23). In this study the experience showed that fear, patient trust in the provider, lack of support for empowerment and exploration patient preferences and lack of appreciation that patients as experts in their disease are some of the reasons that support disempowering paternalistic tendencies.

Enabling interactional dynamics are known to be highly dependent of the socio-cultural context $(9,24)$.

Paternalistic tendencies are quite common in authoritarian cultures where decisions tend to be provider-led and patients assume a passive role (12). In this study, some patients felt that it was inappropriate and socially unacceptable to engage in a dialogue with their provider even when some decision made were not favourable. Poor SES, low medical literacy, fear of being denied services especially where services are free are some of the factors associated passivity in decision making(25). In a Tanzanian study, patients felt that providers were an authority that cannot be questioned and need to be trusted(10). Therefore, in cultural settings such as Malawi 
where social values are inclined towards paternalism and public services are free; it is the providers' responsibility to intentionally tilt the power balances to enable patients for participation.

Patients in this study present both fear and trust with their providers as reason for assuming a passive role in medical decisions. This finding can be validated by a qualitative study done on dialysis patients by Aasen et al who reported that the overbearing provider dominance in medical decisions lead to patients feeling unrecognised and powerless(26). Their study added that; due to the realisation of their powerlessness over decisional control. Consequently, patients felt pushed into a disadvantaged perspective; a situation that obligated them to a submissive stance in order to win the favours of deserved care and feel safe from deliberate mistreatment (26). Thus, patients had no choice but to put their supposed trust in the provider. The cycle of powerlessness and passivity sadly then, gets perpetuated. The reported trust and submission in our study therefore, may not be legitimate, rather emanating out of powerlessness to the provider authority. Further studies are therefore are required to explicate the depicted trust.

\section{Lack of evaluation for suitability of decisions made}

While some patients indicated that they trust provider-led decisions during the interaction, the findings in this study highlight that some decisions are still perceived inappropriate or unacceptable. Without explicitly questioning the provider, some patients reported to fail or choose not to adhere to such care decisions. Thus, highlighting the lack of mutual evaluation of care plans for suitability with individual and context situations. In spite of this theme being small in this study, Serrano et al therefore stresses that the pertinent role of patient participation in decisions making is examining for suitability of care through individualisation and contextualisation of such plans (22). Highlighting that, while being evidence based, care plans need to be coevaluated for alignment to the experience and complex individual situations. This has potential to enhance adherence, concordance and ultimate satisfaction with care.

\section{Limitations Of The Study}

Due to financial reasons, the study was done in the diabetic population thus the experiences may therefore not be generalizable to the general patient population. However, due to the repetitive nature of the contact with the healthcare system, it was felt that DM patients' experiences offers valuable insights into the nature of the health interactions. The narrative in this paper is limited to patients' perceptions and experiences of their involvement in care. Therefore it may fall short of the comprehensive view of the status of patient participation in DM care. However, the patient' insights from patients experiences are equally valuable.

\section{Conclusions}

Though levels vary, it is evident that patients yearn to participate in decisions pertaining to their care. However, the patients' experiences narrated in this paper has unearthed salient gaps associated with the participation in their medical decisions during clinical encounters. While the reasons may be numerous; from individual to contextual issues, it is hoped that it awakens interest practical debate for improvement of patient participation in decision making in chronic care encounters.

Page 12/16 
Even though most Malawian patient- provider encounters are time pressed, providers ought to invest in a little more time to solicit patient involvement in care and use patients' experiential expertise. Providers need a culture change to support patients' participation. To effect this, most importantly, we need to transform providers' medical education. The first step is to review of the medical education curriculum to explore the inclusion and/or effective delivery of competencies and attitudes that value the culture of patient participation. Thus, more implementation studies are needed to elucidate effective ways of delivering such competencies among providers.

\section{Declarations}

\section{Acknowledgements}

We would like to acknowledge the patients who participated in this study as well as the supervisory team.

\section{Funding}

This work was supported by the Consortium for Advanced Research Training in Africa (CARTA). CARTA is jointly led by the African Population and Health Research Centre and the University of the Witwatersrand and funded by the Carnegie Corporation of New York (Grant No-B 8606.R02), Sida (Grant No:54100113), the DELTAS Africa Initiative (Grant No: 107768/Z/15/Z) and Deutscher Akademischer Austauschdienst (DAAD). The DELTAS Africa Initiative is an independent funding scheme of the African Academy of Sciences (AAS)'s Alliance for Accelerating Excellence in Science in Africa (AESA) and supported by the New Partnership for Africa's Development Planning and Coordinating Agency (NEPAD Agency) with funding from the Wellcome Trust (UK) and the UK government.

\section{Conflict of interest}

The authors declare neither financial nor personal interests influencing them whilst engaging with this manuscript.

\section{Authors' contributions}

The first author Martha Makwero (MM) conceptualized the idea with the help of last author Jude Igumbor (JI). Further, MM collected data, analysed the data. The third author Felix Anyanwu (FA) helped with quality and trustworthiness checks. MM drafted the initial manuscript and Adamson Muula (AM), JI and FA contributed to improving the manuscript content and proofreading.

\section{Ethical approval and consent to participate}

The study was approved at both the university of Malawi College of Medicine Research and Ethics Committee (COMREC)study number P.04/19/2654 and the University of the Witwatersrand Human Research Ethics 
Committee (HREC) study number M19550. Written permission to carry out the study was obtained from the relevant hospital authorities and consent obtained from the individual participants. Patient involvement as participants in this study was in accordance with ethical guidelines from the declaration of Helsinki.

\section{Availability of data and materials}

The datasets used and/or analysed during the current study are available from the corresponding author on reasonable request.

\section{Consent for publication}

Not applicable

\section{References}

1. Den Ouden H, Vos RC, Rutten GEHM. Effectiveness of shared goal setting and decision making to achieve treatment targets in type 2 diabetes patients: A cluster-randomized trial (OPTIMAL). Heal Expect. 2017;20(5):1172-80.

2. Tamhane S, Rodriguez-Gutierrez R, Hargraves I, Montori VM. Shared Decision-Making in Diabetes Care. Curr Diab Rep. 2015;15(12):0-10.

3. Wang MJ, Hung LC, Lo YT. Glycemic control in type 2 diabetes: Role of health literacy and shared decisionmaking. Patient Prefer Adherence. 2019;13:871-9.

4. Castro EM, Van Regenmortel T, Vanhaecht K, Sermeus W, Van Hecke A. Patient empowerment, patient participation and patient-centeredness in hospital care: A concept analysis based on a literature review. Patient Educ Couns [Internet]. 2016;99(12):1923-39. Available from: http://dx.doi.org/10.1016/j.pec.2016.07.026

5. Lewis KB, Stacey D, Squires JE, Carroll S. Shared decision-making models acknowledging an interprofessional approach: A theory analysis to inform nursing practice. Res Theory Nurs Pract. 2016;30(1):26-43.

6. Budhwani S. The Self-Management-Focused Chronic Care Model: A Conceptual Framework [Internet]. Toronto: Health system performance network; 2013. p. 1-16. Available from: w w w . i h p m e . u t o r o n t o. c a

7. Shortus T, Kemp L, Mckenzie S, Harris M. "Managing patient involvement”: Provider perspectives on diabetes decision-making. Heal Expect. 2013;16(2):189-98.

8. World Health Organization (WHO). Delivering quality health services; A global imperative for universal health coverage [Internet]. Switzerland: OECD, and International Bank for Reconstruction and Development/The World Bank; 2018. 1-200 p. Available from: http://apps.who.int/iris.

9. Peek ME, Wilson SC, Gorawara-Bhat R, Odoms-Young A, Quinn MT, Chin MH. Barriers and facilitators to shared decision-making among African-Americans with diabetes. J Gen Intern Med. 2009;24(10):1135-9. 
10. Vedasto O, Morris B, Furia FF. Shared Decision-making between health care providers and patients at a Tertiary Hospital Diabetic Clinic in Tanzania. 2020;1-8.

11. Elwyn G, Edwards A, Wensing M, Hood K, Atwell C, Grol R. Shared decision making: Developing the OPTION scale for measuring patient involvement. Qual Saf Heal Care. 2003;12(2):93-9.

12. Peek ME, Odoms-Young A, Quinn MT, Gorawara-Bhat R, Wilson SC, Chin MH. Race and shared decisionmaking: Perspectives of African-Americans with diabetes. Soc Sci Med [Internet]. 2010;71(1):1-9. Available from: http://dx.doi.org/10.1016/j.socscimed.2010.03.014

13. Cubaka VK, Schriver M, Kayitare JB, Cotton P, Maindal HT, Nyirazinyoye L, et al. He should feel your pain. Africa J Prim Healthc Fam Med. 2081;1(4):1-11.

14. Ekdahl AW, Andersson L, Wiréhn AB, Friedrichsen M. Are elderly people with co-morbidities involved adequately in medical decision making when hospitalised? A cross-sectional survey. BMC Geriatr [Internet]. 2011;11(1):46. Available from: http://www.biomedcentral.com/1471-2318/11/46

15. Serrano V, Rodriguez-Gutierrez R, Hargraves I, Gionfriddo MR, Tamhane S, Montori VM. Shared decisionmaking in the care of individuals with diabetes. Diabet Med. 2016;33(6):742-51.

16. Joann Seo, Melody S. Goodman, Mary Politi, Melvin Blanchard KA. Effect of Health Literacy on DecisionMaking Preferences among Medically Underserved Patients. PMC. 2016;36(4):550-6.

17. Guillermo Garcia-Manero, Hui Yang, Shao-Qing Kuang, Susan O’Brien, Deborah Thomas and HK. Patient Activation and Advocacy: Which Literacy Skills Matter Most? J Health Commun [Internet]. 2011;16(3):17790. Available from: https://www.ncbi.nlm.nih.gov/pmc/articles/PMC3624763/pdf/nihms412728.pdf

18. Bailey RA, Shillington AC, Harshaw Q, Funnell MM, VanWingen J, Col N. Changing Patients' Treatment Preferences and Values with a Decision Aid for Type 2 Diabetes Mellitus: Results from the Treatment Arm of a Randomized Controlled Trial. Diabetes Ther [Internet]. 2018;9(2):803-14. Available from: https://doi.org/10.1007/s13300-018-0391-7

19. Lyles CR, Schillinger D. Patient-provider communication and diabetes medication adherence: where do we go from here? Diabetes Manag. 2013;3(3):185-8.

20. Podlog LW, Brown WJ. Self-determination Theory: A Framework for Enhancing Patient-centered Care. J Nurse Pract [Internet]. 2016;12(8):e359-62. Available from:

http://linkinghub.elsevier.com/retrieve/pii/S1555415516301131

21. Da Silva D. Helping people share decision making [Internet]. The Health Foundation. 2012. 1-13 p. Available from: http://www.health.org.uk/public/cms/75/76/313/3448/HelpingPeopleShareDecisionMaking.pdf? realName=rFVU5h.pdf

22. Armstrong MJ, Shulman LM, Vandigo J, Mullins CD. Patient engagement and shared decision-making: What do they look like in neurology practice? Neurol Clin Pract [Internet]. 2016;6(2):190-7. Available from: http://www.ncbi.nlm.nih.gov/pubmed/27104070\%0Ahttp://www.pubmedcentral.nih.gov/articlerender.fcgi? artid=PMC4828680

23. Martos-Méndez MJ. Self-efficacy and adherence to treatment: the mediating effects of social support. J Behav Heal Soc Issues [Internet]. 2015;7(2):19-29. Available from:

http://dx.doi.org/10.5460/jbhsi.v7.2.52889 
24. Peek ME, Lopez FY, Williams HS, Xu LJ, McNulty MC, Acree ME, et al. Development of a Conceptual Framework for Understanding Shared Decision making Among African-American LGBT Patients and their Clinicians. J Gen Intern Med [Internet]. 2016;31(6):677-87. Available from: http://dx.doi.org/10.1007/s11606-016-3616-3

25. Guillermo Garcia-Manero, Hui Yang, Shao-Qing Kuang, Susan O'Brien DT. Patient Activation and Advocacy: Which Literacy Skills Matter Most? J Health Commun [Internet]. 2011;23(1):1-7. Available from: https://www.ncbi.nlm.nih.gov/pmc/articles/PMC3624763/pdf/nihms412728.pdf

26. Aasen EM, Kvangarsnes M, Heggen K. Perceptions of patient participation amongst elderly patients with end-stage renal disease in a dialysis unit. Scand J Caring Sci. 2012;26(1):61-9.

\section{Supplementary Files}

This is a list of supplementary files associated with this preprint. Click to download.

- InterviewguideforexplorationofPatientcentredcare.docx 